\title{
Psychosocial problems of schoolchildren and the psychological counseling approaches implicated by counselors for treating it
}

\author{
Mohammad Qassim Abdullah \\ University of Aleppo, Syria \\ *)Corresponding author, Đe-mail: mk.abdalah@yahoo.com
}

\begin{abstract}
This study aims to examine and evaluate the behavioral problems of schoolchildren in basic education and the psychological counseling approaches utilized by counselor for its treatment. It also assesses the differences in these approaches in accordance with their gender and educational qualification/degree. The sample consists of (240) counselors (125 males, 115 females) enrolled from five educational administrations with the concluded scale used to obtain data due to its high level of validity and reliability. The findings showed that the most common behavioral problems were disobedience, teachers' underachievement, and aggression. In addition, the most common approaches used by counselors were individual and group counseling, Leisure time counseling, rational-emotive therapy, plying counseling, reality therapy, and client-centered therapy. The result showed that there were no significant differences in counseling approaches relating to gender and academic qualification of counselors, except for the approaches of cognitive, behavioral and group counseling techniques.
\end{abstract}

Keywords: psychosocial problems, counseling approaches, schoolchildren

How to Cite: Abdullah, M.Q. (2017). Psychosocial problems of schoolchildren and the psychological counseling approaches implicated by counselors for treating it. Couns-Edu: International Journal of Counseling and Education, 2(4): pp. 150-159. DOI: https://doi.org/10.23916/0020170212940

This is an open access article distributed under the Creative Commons Attribution License, which permits unrestricted use, distribution, and reproduction in any medium, provided the original work is properly cited. (C2017 by author and Indonesian Counselor Association (IKI).

\section{Introduction}

The continuous increase in technological advancement in the present era tend to pressurize children to break up with their families,leads to a rapid sociocultural paradigm, and a rise inbehavioral problems that are also similarly steep and disrupting.

Children and teens seem irritable or even hostile when they are tired or ill. They tend to argue or disobey their parents in a bid to show maturity. In addition, young children sometimes lie to get out of trouble because they do not understand the differences with truth. Although this seems normal, however, when they act this way each time they get into trouble at home, school, or with other kids in the neighborhood, they are likely to possess a disruptive behavior disorder(Prakash, Mitra, \& Prabhu, 2008).

Knowledge of child mental-health problems is important due to its interference with social and educational development, thereby, capable of saving life-long psychiatric problems (Rutter, 1996). There is a little consensus on the extent to which manifestations of psychiatric symptoms are universal and affected by cultural aspects. However, since studies have found prevalence rates to vary all over the world, it is necessary to consider culture and context in determining the way in which children's psychopathology is manifested (Canino \& Alegría, 2008; R. Hackett \& L. Hackett, 1999; Roberts, Attkisson, \& Rosenblatt, 
1998). There is also evidence that child disorders may vary between developed and developing countries, therefore, making it necessary to distinguish studies by each country(Fleitlich-Bilyk \& Goodman, 2004; R. Hackett \& L. Hackett, 1999). A major gap in the epidemiology of child mental problems is the lack of studies regarding the date on the prevalence rates in low-middle-income countries (Kieling et al., 2011; Rohde, 2011; Giel, R., Arango, Climent, Harding, Ibrahim Ladrito-Ignacio, Sriniasa, Salazar, Wigand Younis, 1981).

The vulnerability of primary schoolschildren to infection, is similar their adoptative behavior in this age group. However, it remains a cause of concern to their parents till they are become adults. Many times parents do not bother on such habits because they are certain of repentance in the nearest future. Language and speech disorder are in school-age population are more prevalent in toddlers and pre-school with low morbidity in habit disorder and behaviorial problems, which keeps them away from friends due to feeling of guilt (Anderson, 2012). There are two main types of disruptive behavior namely: Oppositional Deviant Disorder (ODD) and Conduct Disorder (CD). Extensive review of issues pertaining to the relationship between externalizing behavior problems and academic underachievement stated that, in children, inattention and hyperactivity were strongly correlatedwith academic problems than aggression, (Eimani-Oshnari1, Amiri-Majd, Babakhany,2014, Anderson,2012).

Estimates of the number of school-aged children with emotional problems vary. For instance the incidence and prevalence figures were controversial, with estimates ranging from $2-3 \%$ to $22 \%$ (Fleisch, \& Goodman 2000; Lund, 2008). The numbers increased when those referred to as at risk are included (Lavin, Korte, \& Davies, 2011). Review of recent studies concentrating on mental-health problems among school children showed the prevalence figures varied from $6.33 \%$ to $43.1 \%$ in Indian context (1-6), which was similar to the International variability. (7- 10) The available literature also speaks of variability and inadequacies of diagnostic methods, research criteria, treatment modalities and psychosocial interventions, (Prakash, Mitra, \& Prabhu, 2008).

According to studies, there are a considerable number of youngsters with false-positive misdiagnosed disabilities/disorders. Therefore, schools have the ability to accomplish their goal of teaching only when they have addressed the psychosocial problems that interfere with students' learning. In schools, youngsters with serious emotional and learning problems are assisted under the auspices of "special education."However, many students with behavior, learning, and emotional problems do not meet this criteria, therefore, their needs need to be addressed through support programs and other accommodations, especially psychological counseling programs in schools, (Fleitlich \& Goodman, 2000).

Saurl and Loureiro (2014) investigatedthe mental-health, behavioral and emotional problems, in a cohort of schoolchildren in accordance with their gender and behavioral performance to the family. Data was obtained from 677 children in Ribeirão Preto (SP), Brazil, between the ages of 10/11. The mental health assessment was performed using the Brazilian version of the Strengths and Difficulties Questionnaire. The results showed that the prevalence rates for boys and girls were $41.7 \%$ and $34.5 \%$ for total difficulties score, $50.4 \%$ and $57.6 \%$ for emotional symptoms, $31.2 \%$ and $18.8 \%$ for hyperactivity, $38.8 \%$ and $27.6 \%$ for conduct problems, $27.1 \%$ and $26.7 \%$ for peer problems and, $4.7 \%$ and $2.7 \%$ for prosocial behavior, respectively. The family characteristics associated with behavioral problems were low socioeconomic status and poor maternal education for boys and girls (Deivasignamini, 1990; Sakar, Kapur,\& Kaliaberumal 1996).

A research conducted by Anderson on Jamaican children showed that they suffered many times from psychosocial and handicapping conditions such as a lack of understanding and empathy from teachers, administrators and their peers. This literature is replete with evidence of such treatment leading to negative student behavior and the resultant of poor performance. Therefore, this is consistent with the fact that the number of students with special needs or learning difficulties haverapidly increased.However, the ones most likely to be misunderstood are those with emotional and behavioral disorder. This paper questions the issued associated with what researchers consider normal behavior in schools. A discussion of the construct "emotional and behavioral disorders" within the purview of the Jamaican educational system is provided in order to give credence and validity to this research. Intervention/Treatment models are offered followed by practical and evidenced-based classroom strategies addressing the needs of students with emotional and behavior disorders. The aforementioned also supports the researchers' view that changes 
are long overdue in creating standards and significant guidelines for helping students with emotional and behavioral problems(Roberts, Attkisson, \& Rosenblatt, 1998).

There is a significant rise in the behavioral problems in childrenwith various literatureson the variability and inadequacies of diagnostic methods, research criteria, treatment modalities and psychosocial interventions. Prakash, Mitra, and Prabhu (2008), carried out a research to assess the behavior problems of50 children between the ages of 6-14 years attending government school. They were selected after appropriate randomization and subsequently assigned to Child behavior checklist. The data collected was suitably interpreted using appropriate statistical tests and the results showed that Twenty-one (42\%) children were above the cut-off score with amean CBCL score of 43.3. Furthermore, the most common behavior problems in these subjects namely "cannot sit still, restless, hyperactive" were shown by $62 \%$ of the subjects. Female children had behavior problems such as "too concerned with neatness or cleanliness", "self-conscious or easily embarrassed" and "feelings of perfection" while male children had behavior problems such as "Does not feel guilty after misbehaving", "inability to concentrate" and "restless." In conclusion, the analysis of pattern of distribution of behavior problems in the subjects showed that female children possessed more internalizing behavior problems. (Prakash, Mitra, \& Prabhu, H.R. 2008, Abdullah, 2016).

Syria is a developing country witha large population, consisting of a significant proportion of children attending schools. Therefore, the behavioral problems and psychiatric syndromes in the children are a matter of concern because its consequences tends to seriously impair their ability to become useful and productive citizens in future.The vulnerability of children increasesdue tothe unavailability of effective parenting. This study aim to assess the prevalence of behavioral problems among school children. The research findings helpsto determine the prevalence, associated Factors and predictors that are useful in early diagnosis and management. Reviews on the diverse method of data collection and diagnosis carried out in this field revealed that the prevalence of behavior problems in children is high (Abdullah, 1992).

Understanding the root cause of the child's behavior is essential, because it helps to stabilize the behavior strategy (Tolan, and Gorman-Smith, 2001; Abdullah, 2012). There are five basic models in understanding child behaviornamely biological, behavioral, Cognitive, systemic, and psychodynamic. The systemic approach targets the individual's behavior within the systemsuch as their family, friends, school, classmates, community, and clubs (Abdullah, 2016, Anderson, 2012).

The counselors need to use many techniques and approaches to conduct programs that are dependent on the theories of counseling and psychotherapy such as Reality therapy, client-centered counseling, group therapy, behavioral counseling, systematic, and family. Hence, this study focuses on investigating the behavioral problems of schoolchildren and their counselors for treating these problems.

\section{Objectives of the study}

This research aimed to examine the following:

1. The most common behavioral problems among schoolchildren.

2. The most common psychological counseling programs and techniques have been applicated in the schools.

3. The differences in implication counseling techniques regarding gender, and academic qualification of counselors.

\section{Questions of the study}

This research addressed the following three questions:

1. What are the most common behavioral problems among schoolchildren?

2. What are the most common psychological counseling programs and techniques have been implicated by counselors in the schools.

3. What are the differences in implication counseling techniques regarding the variables of gender, and academic qualification of counselors? 


\section{Method}

Participants: The total population of the counselors in the formal educational schools consist of a total of 3500 psychological counselors, and 1500 social counselors from the Department of Educational Researches in Ministry of Education. Participants of the study include 240 counselors consisting of 125 males, and 115 females enrolled from five cities in Syria namelyDamascus, Homs, Hama, Lataqia, and Der Al-Zoor.

Procedure: The instrument used for the study was questionnaire divided into two parts. The first, consists of 50-items used to scale the behavioral problems of school children from the perspectives of their counselors, in addition to one open question at the end of this division. The second part consists of 20items relating to psychological counseling techniques/theoretical approaches used by counselors, in addition to an open question on the approaches not mentioned in the scale.

The instrument evaluates behavioral (emotional, social, cognitive) problems regarding the classroom behavior, learning-teaching, and group participation such as Underachievement, teacher's mistreatment, attention problems, anti-social behaviors, sexual deviation, food disorders, obsessive compulsive disorders, sleep disorders , disruptive behavior, aggression, loneness, test anxiety, shyness, anger, and learning difficulties. Reliability and validity of this scale are 0.89 , while the Cronbach's alpha coefficient is 0.86 and for subscales varies from 0.79 to 0.89 .

Data collection and analysis

Descriptive statistics (percentages, M, SD), and T-test are used to assess the significance of differences regarding the counselor's gender variable. In addition, thefisher-test was used to assess the differences in psychological counseling approaches implicated bycounselors regarding the academic qualifications/degree variable.

\section{Results and Discussions}

Table 1 is used to answer the first question: "What are most common behavioral problems among school's children."

Table 1 the highest common behavioral problems of children and means

\begin{tabular}{lll}
\hline & The behavioral problems & Mean \\
\hline $\mathbf{1}$ & disobedience of teachers & 4.35 \\
$\mathbf{2}$ & underachievement & 4.31 \\
$\mathbf{3}$ & Aggressive behaviors & 4.26 \\
$\mathbf{4}$ & Delaying the academic tasks & 4.13 \\
$\mathbf{5}$ & Repeated absence of school & 3.97 \\
$\mathbf{6}$ & Teacher's maltreatment & 3.85 \\
$\mathbf{7}$ & Difficulties with teachers & 3.71 \\
$\mathbf{8}$ & Loneness & 3.66 \\
$\mathbf{9}$ & Test anxiety & 3.57 \\
$\mathbf{1 0}$ & Shyness & 3.45 \\
$\mathbf{1 1}$ & Anger and impulsiveness & 3.14 \\
$\mathbf{1 2}$ & Learning disabilities & 2.98 \\
\hline
\end{tabular}

Table (1) shows that the most common behavioral problems of children in schools were: disobedience to teachers $(M=4.35)$, underachievement $(M=4.31)$, aggressive behaviors $(M=4.26)$, delaying the academic tasks $(M=4.13)$, repeated absence from schools $(M=3.97)$, teacher's maltreatment $(M=3.85)$, 
difficulties with teachers $(M=3.71)$, and loneness $(M=3.66)$. Table 2 shows the test anxiety, shyness, anger, impulsiveness, and learning disabilities of children.

Table 2 the lowest behavioral problems of children and mean

\begin{tabular}{lll}
\hline & Behavioral problems & Mean \\
\hline $\mathbf{1}$ & Obsessive-compulsive problems & 1.95 \\
$\mathbf{2}$ & Bedwetting ( Enuresis) & 1.91 \\
$\mathbf{3}$ & Sexual deviation & 1.85 \\
$\mathbf{4}$ & Eating (food) problems & 1.67 \\
$\mathbf{5}$ & Sleep problems & 1.35 \\
$\mathbf{6}$ & Hyperactivity & 1.27 \\
$\mathbf{7}$ & lie & 1.17 \\
$\mathbf{8}$ & Psychosis syndromes & 1.11 \\
\hline
\end{tabular}

Table 2 shows the lowest problems consecutively such as obsessive-compulsive problems, bedwetting, sexual deviation, eating problems, sleep problems, hyperactivity, and psychosis syndromes.

Table 3, answers the second question "what are the most common psychological approaches used by counselors for treating these problems."

Table 3Psychological counseling approaches implicated by counselors

\begin{tabular}{lll}
\hline & Psychological counseling approaches/techniques & Mean \\
\hline $\mathbf{1}$ & Individual counseling & 4.87 \\
$\mathbf{2}$ & Group therapy & 4.74 \\
$\mathbf{4}$ & Leisure time counseling & 4.68 \\
$\mathbf{5}$ & Clint-centered therapy & 4.63 \\
$\mathbf{6}$ & Systematic counseling & 4.53 \\
$\mathbf{7}$ & Behavioral counseling & 4.48 \\
$\mathbf{8}$ & Cognitive therapy & 4.35 \\
$\mathbf{9}$ & Plying counseling approach & 4.15 \\
$\mathbf{1 0}$ & Brief therapy & 3.93 \\
$\mathbf{1 1}$ & Rational-Emotive therapy & 3.75 \\
$\mathbf{1 2}$ & Psycho-analytic therapy & 3.65 \\
$\mathbf{1 3}$ & Reality therapy & 3.47 \\
$\mathbf{1 4}$ & Traits-factors approach in counseling & 3.41 \\
$\mathbf{1 5}$ & Logo therapy \& existential counseling & 3.21 \\
$\mathbf{1 6}$ & Gestalt psychotherapy & 3.13 \\
$\mathbf{1 7}$ & Eclectic psychotherapy & 3.09 \\
$\mathbf{1 8}$ & Egan's Effective helping counseling & 2.85 \\
\hline & Family counseling approach & 2.78 \\
\hline
\end{tabular}


Table 3 shows the most common counseling approaches used by counselors for treating children's problems such as Individual counseling, Group therapy, Leisure time counseling, Clint-Centered therapy, Systematic counseling, and Behavioral counseling. While the latest counseling techniques have been implicated were logo therapy \& existential counseling, Gestalt psychotherapy, Eclectic psychotherapy, Egan's Effective helping counseling and finally, and family counseling approach.

Regarding the third question "What are the differences in implication counseling techniques regarding the variables of gender, and academic qualification of counselors," The T-test was used to evaluate the counseling approach between male and female of counselors. Table 4 shows the results of this statistical analysis.

Table 4 the differences in application of counseling approaches regarding the gender variable of counselors

\begin{tabular}{|c|c|c|c|c|c|}
\hline Counseling technique & $\mathbf{N}$ & & M SD & T.value & Sig. \\
\hline \multirow[t]{2}{*}{ Rational-emotive therapy } & 120 male & 2.65 & 0.76 & -2.52 & 0.016 \\
\hline & 115 female & 3.14 & 0.98 & & \\
\hline \multirow[t]{2}{*}{ Reality therapy } & 124 male & 3.12 & 0.32 & -2.48 & 0.081 \\
\hline & 115 female & 3.76 & 0.17 & & \\
\hline \multirow[t]{2}{*}{ Plying counseling approach } & 125 male & 2.11 & 1.08 & -2.18 & 0.043 \\
\hline & 115 female & 2.57 & 1.19 & & \\
\hline \multirow[t]{2}{*}{ Client-Centered therapy } & 125 male & 3.87 & 0.48 & -2.69 & 0.0127 \\
\hline & 114 female & 3.21 & 0.19 & & \\
\hline \multirow[t]{2}{*}{ Brief therapy } & 125 male & 3.09 & 0.56 & -2.53 & 0.0142 \\
\hline & 115 female & 2.56 & 0.87 & & \\
\hline \multirow[t]{2}{*}{ Multiple counseling } & 125 male & 3.45 & 0.24 & -2.49 & 0.02 \\
\hline & 115 female & 3.19 & 0.57 & & \\
\hline
\end{tabular}

Table 4 shows the most important counseling approaches/techniques used by school female counselors namely Rational-Emotive Therapy $(\mathrm{T}=-2.52)$, Reality Therapy and plying counseling approaches $(\mathrm{T}=$ $2.48,-2.18)$, while the most important counseling approaches implicated by male counselors are ClientCentered therapy $(T=-2.69)$, Brief therapy $(T=-53)$, and Systematic counseling approaches $(T=-2.49)$. 
For the differences in implication counseling approaches regarding counselor's academic qualification or degrees, the results of "Fisher" test, are shown in table 5.

Table 5 The differences in application of counseling approaches regarding the academic qualification/degree variable of counselors

\begin{tabular}{llcrl}
\hline Counseling approach & Academic Qualification & Mean & $\begin{array}{c}\text { Calculated } \\
\text { differences }\end{array}$ & $\begin{array}{l}\text { Critical } \\
\text { Value }\end{array}$ \\
\hline Cognitive Therapy & Bachelor + Diploma & $(2.21)(2.71)$ & -0.52 & 0.467 \\
& Bachelor + Master & $(2.21)(2.63)$ & -0.42 & $0.521^{*}$ \\
& Diploma + Master & $(2.73)(2.54)$ & -0.41 & 0.486 \\
Behavioral therapy & Bachelor + Diploma & $(3.14)(3.85)$ & -0.71 & $0.04^{*}$ \\
& Bachelor +Master & $(3.20)(3.78)$ & -0.58 & 0.38 \\
Group therapy & Diploma + Master & $(3.63)(3.95)$ & -0.32 & $0.45^{*}$ \\
& Bachelor + Diploma & $(2.45)(2.92)$ & 0.53 & $0.64^{*}$ \\
& Bachelor +Master & $(2.44)(2.69)$ & -0.24 & 0.43 \\
& Diploma + Master & $(2.43)(2.86)$ & -0.44 & $0.03^{*}$ \\
\hline
\end{tabular}

* sig. 0.05

Table (5) shows that there are significant differences between the bachelor and master degree in cognitive therapy, with the master qualification higher $(\mathrm{M}=2.63)$ than bachelor $(\mathrm{M}=2.21)$. In addition the behavioral therapy among diploma $(\mathrm{M}=3.85)$ is higher than bachelor $(\mathrm{M}=3.14)$, and between master higher $(M=3.78)$ than bachelor $(M=3.20)$, and among master higher $(M=3.95)$ than among diploma $(M=3.63)$. Group therapy has been used by counselor with diploma higher $(M=2.92)$ than among those getting bachelor degree $(M=2.45)$, and higher among master degree $(M=2.86)$ than among diploma degree $(\mathrm{M}=2.43)$.

\section{Discussion}

The results showed that behavioral problems of schoolchildren were vicarious. The higher prevalence problems were noncompliance, low school achievement, and aggression, in contrast to the problems of obsessive-compulsive, enuresis, sexual deviation and lying. These problems reflect the conduct disorder in childhood, and it needs to be analyzed in perspective of the context and environment of children inside and outside the school, especially in family. Conversely, it has been evident that disorders are not different from the view of fathers, mothers, and teachers (Eimani-Oshnari, Mojtaba Amiri-Majd Babakhany, 2014). Moreover, teachers evaluated more anxiety problems than parents. The above findings explained by the intensity of hyperactivity symptoms and problems in schools as well as homes being the probable causes for visiting counselors for them to observe the symptoms in both environments. The cognitive and emotional problems are affected by learning problems due to lack of attention, and is rejected by classmates due to their immature behavior. The Results of this research are consistent with the studies of Weiler et al (1999), and Saur and Loureiri (2014). Important to direct attention to the point that the confidence associated with children problems dependent on the reports of counselors, parents, teachers and doctors for diagnosing and designing counseling programs. Some behavioral problems are situational to an environment such as a home or school, or some disorders ignored because they are not problematic. Background check indicated that the grading scales are used in different cultures, (Kafle, Vaidya, Panta, Chhetri,M. and Mehrotra, 2010).

According to the counselor's gender variable, the most important approaches/techniques used by school female counselors are: rational-emotive therapy, reality therapy and plying counseling, while those 
implicated by male counselors are: client-centered therapy, brief therapy, and systematic counseling approaches. Conversely, the finding showed significant differences between counselors with bachelor and master degree in cognitive therapy. In addition to behavioral therapy between diploma and bachelor, is higher than diploma. This finding reflecting the experience and professional development of counselor's skills that meet the demands and the variety of programs need to be conducted to treat the behavioral problems in schools. It is very important for males and females counselor to depend on integration trend in designing counseling programs, to meet the developmental demands and tasks of childhood (Abdullah, 1992). Therefore, the theoretical background represents the foundation for succession counseling programs in schools (Poulou, and Norwich, 2000).

\section{Conclusions and Recommendations}

This study examined behavioral problems of schoolchildren and the counseling approaches used for its treatment. These problems influence mental health of children and their ability to adjust in school. In addition, it investigated the theoretical counseling approaches the have been implicated for its treatment, and the differences in applying these approaches regarding the variables of gender and qualification or academic degree of the counselors.

The finding revealed that the higher prevalence problems were noncompliance, low school achievement, and aggression, in contrast to the obsessive-compulsive, enuresis, sexual deviation and lying that were minimal. On the other hand, the most common counseling approaches used by counselors for treating children's problems are as follows Individual counseling, group therapy, leisure-time counseling, client-centered therapy, systematic counseling, and Behavioral counseling. While the latest counseling techniques were logo therapy \& existential counseling, gestalt psychotherapy, eclectic psychotherapy, Egan's effective helping counseling and finally, family counseling approaches. The findings indicated the significant differences regarding the academic qualification and degree utilized by counselors. Hence, these significant differences found between counselors with a bachelor and master degree in cognitive therapy. In addition, behavioral therapy conducted by counselors with a diploma were greater than those with bachelor degree, and among counselors with master more than with bachelor and among counselors with master higher than among with diploma. Counselors with diploma have been used group therapy more than those with bachelor degree, and masters. According to the counselor's gender variable, the most important counseling approaches/techniques used by school female- counselors are: rational-emotive therapy, reality therapy and plying counseling approaches, while the most important counseling approaches implicated by male- counselors are: client-centered therapy, brief therapy, and systematic counseling approaches.

These findings lead to significant recommendations:

1) Further research can be conducted to explore the behavioral problems according to developmental stages in childhood and adolescence.

2) Examine the differences in application counseling approaches and techniques among schoolpsychological counselors according to demographic variables.

3) Another issue that needs to be further studied within informing science research is the need to use other methodology such as the developmental longitudinal research, experimental method to highlight the "cause-result" hypothesis and to explore causal factors of these complex behavioral problems, in addition to the relationship between children's mental health and school environment.

4) Incorporate a variety of counseling strategies, methods and experiences for counselor's skills developmental programs. A more efficient instructional delivery system result from skills assessment of specialists seeking enhancement or development of academic practice and experiences.

\section{Acknowledgments}

The author is grateful to the Education College at Aleppo University for reviewing the instrument of the research, and to psychological counselors for their encouragement and application the scales of the research. 


\section{References}

Abdullah, Q. M. (1992).The group differences in help-seeking behavior among adolescents. Acta Paedopsychiatrica, International Journal of Child and Adolescent Psychiatry. (55), 4, 223-227.

Abdullah, Q. M. (2012). Counseling and psychotherapy theories. University of Aleppo Press.

Abdullah, Q. M. (2016). Introduction to mental health. Dar Alfikr: Amman.

Anderson, S. A. (2012). Psycho-Educational Processes as Strategies for Students Presenting with Emotional and Behavioral Disorders American International Journal of Contemporary Research Vol. 2 No. 7, 25-34.

Canino, G., \& Alegría, M. (2008). Psychiatric diagnosis - is it universal or relative to culture? Journal of Child Psychology and Psychiatry, 49(3), 237-250.

Deivasignamini T., R. (1990).Psychiatric morbidity in primary schoolchildren. An epidemiological study. Indian J Psychiatry; 32, 235-40.

Eimani-Oshnari1, M. Amiri-Majd, M., Babakhany,V. (2014). A Comparison of emotional and behavioral problems in children with ADHD at home and school. Eimani-Oshnari M., et al., J Anal Res Clin Med, 2(2), 64-70.

Fleitlich-Bilyk, B., \& Goodman, R. (2004). Prevalence of child and adolescent psychiatric disorders in southeast Brazil. Journal of the American Academy of Child \& Adolescent Psychiatric, 43(6), 727-734.

Fleitlich, B., \& Goodman, R. (2000). Epidemiologia. Revista Brasileira de Psiquiatria, 22(Sup1.2), 2-6.

Giel, R., Arango, M. Climent, C., Harding, T., Ibrahim, H. Ladrito-Ignacio,L., Sriniasa, R., Salazar, M., Wig, N. and Younis, Y. (1981). Childood mental health disorders in primary health care: results of observations in four developing countries, Pediatrics, 68, (5), 677-683.

Hackett, R., \& Hackett, L. (1999). Child psychiatry across cultures. International Review of Psychiatry, $11,225-235$.

Kafle P. Vaidya, L., Panta, P. Chhetri, M. and Mehrotra, S. (2010). Common behavior problems amongst primary school children in slum dwelling area of Kathmandu Valley Nepal. Med Coll Journal, 12 (3), 187-189.

Kieling, C., Baker-Henningham, H., Belfer, M., Conti, G., Ertem, I., Omigbodun, O., Rohde, L. A., Srinath, S., Ulker, N., \& Rahman, A. (2011). Child and adolescent mental health worldwide: Evidence for action. The Lancet, 378(9801), 1515-1525.

Lavin, A., Korte, L. \& Davies, T. (2011). The impact of classroom technology. Journal of Journal of Technology Research 2, 65-77.

Lund, C., Breen, A., Flisher, A. J., Kakuma, R., Corrigall, J., Joska, J. A., Swartz, L., \& Patel, V. (2010). Poverty and common mental disorders in low and middle-income countries: A systematic review. Social Science \& Medicine, 71(3), 517-528.

Prakash, J., Mitra, A. K. \& Prabhu, H.R. (2008) Child and Behavior: A School Based Study, Delhi Psychiatry Journal, Vol. 11 No.1, 79-82.

Roberts, R. E., Atkinson, C. C., \& Rosenblatt, A. (1998). Prevalence of psychopathology among children and adolescents. American Journal of Psychiatry, 155(6), 715-725.

Rohde, L. A. (2011). The need of epidemiological data on child mental disorders from low-middle income countries. European Child and Adolescent Psychiatry, 20(10), 497-498.

Poulou, M., and Norwich, B. (2000) Teachers' causal attributions, cognitive, emotional and behavioral responses to students with emotional and behavioral difficulties, British Journal of Educational Psychology, 70, 559-581.

Rutter, M. (1996). Connections between child and adult psychopathology. European Child and Adolescent Psychiatry, 5(Suppl. 1), 4-7.

Saur, A. and Loureiri, S. (2014) Behavioral and emotional problems of schoolchildren according to gender. Arquivos Brasileiros de Psicologia; Rio de Janeiro, 66 (1): 102-116. 
Sakar A.B, Kapur M, Kaliaberumal V.G. (1996) The prevalence and pattern of psychological disturbance in school going middle childhood children. Natl Insti Mental Health Neuro Sci (NIMHANS) J.; 13, 33-41.

Tolan, P. and Gorman-Smith, D. (2001). New study to focus on efficacy of "whole school" Prevention approaches. Emotional \& Behavioral Disorders in Youth, 2, 5-6, $22-23$.

Weiler M.D., Bellinger D., Marmor J, Rancier S, Waber D.(1999). Mother and teacher reports of ADHD symptoms: DSM-IV Questionnaire data. J Am Acad Child Adolescent Psychiatry 1; 38(9): 1139-47. 